\title{
Le rôle de la conception et de la gestion de la voirie dans la réduction des rejets urbains de temps de pluie
}

\author{
par Jean-Daniel Balades, Pierre Berga \\ Centre d'Etudes Techniques de l'Equipement du Sud-Ouest (C.E.T.E.) \\ Groupement Aquitain pour la Recherche et l'Innovation en Hydrologie (GARIH)*
}

\section{INTRODUCTION}

Les divers usages de la voirie urbaine produisent une accumulation de sédiments qui, lorsqu'ils sont, malgré le nettoyage des voiries, réentraînés par le ruissellement de temps de pluie, contribuent à une pollution non négligeable du milieu récepteur. Des chiffres, quelquefois contradictoires, ont été publiés et on peut retenir les ordres de grandeur suivants :

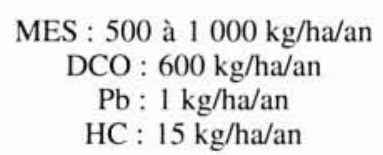

Cette source de pollution étant de nature à affecter les usages de l'eau, il est impératif de la limiter. C'est une préoccupation majeure du GARIH et du réseau technique de l'Equipement sous l'impulsion du LCPC (Laboratoire Central des Ponts et Chaussées). Le CETE du Sud-Ouest, en partenariat avec la CUB (Communauté Urbaine de Bordeaux) s'est donc particulièrement intéressé à deux voies possibles de progrès. Elles sont caractérisées par le besoin d'intervenir sur la pollution le plus en amont possible, au plus près de sa source :

(*) Le GARIH est un Groupement d'Intérêt Scientifique qui comprend sept organismes : l'Agence de l'Eau, le CEMAGREF, le CETE, la CUB, I'IEEB. la Lyonnaise des Eaux et l'Université de Bordeaux 1.

\section{The role of the conception and management of the road network} in the reduction of urban pollutants by rainy weather

The streaming on the urban road network is one of the principal water pollution causes. The deposit and the streaming processes during the rainy episodes must be better known if we want to intervene efficiently against this pollution.

The experimental site on road Roustaing in Talence corresponds to a very urbanized reservoir slope of 4 ha whose urban network supports heavy traffic. The equipment installed there permits a continuous measurement of the rain and the evolution of the pollution streaming on the pavement. At the same time, the deposit accumulation on the road is measured in order to know its variability in accordance with the time interval between two cleanings.

Another approach consists of reducing the streaming. The draining trench is one of the techniques employed for this. Its efficiency is tested on a road in an activity zone which comprises heavy traffic. The quantitative and qualitative aspects are the following : continuous measurement of the rain and its flow, measurement of the water pollution at the end of the trench, migration possibility of the pollution to the ground.

An intervention at the source by an optimisation of the road cleaning (frequency and machine type) or by $a$ better management of the flow will permit a considerable limitation of the impact of urban pollutants by rainy weather. 
- le revêtement de chaussée et son entretien : il est possible d'agir pour améliorer l'organisation du nettoyage et les matériels utilisés, mais aussi pour faciliter le captage à la source même de la pollution avec une conception adaptée de la voirie (choix de la structure et du revêtement de chaussée, choix de la morphologie et notamment de la configuration trottoir/caniveau/chaussée) ;

- les dispositifs de rétention de la pollution au plus près du ruissellement : tranchées, puits, fossés et dispositifs de rétention et de traitement.

Pour mener l'action du GARIH dans ces deux voies, le CETE du Sud-Ouest a installé des équipements de mesures et entamé une campagne d'expérimentation sur deux bassins versants urbains de la Communauté Urbaine de Bordeaux. Il s'agit des sites de :

- la rue Roustaing, à Talence, pour étudier le rôle de l'entretien des voiries,

- l'avenue Gustave Eiffel, à Pessac, pour caractériser les performances hydrauliques et épuratoires d'une tranchée et pour y observer les transferts éventuels de pollution.

\section{II $\square$ LE RÔLE DE LA GESTION ET DE L'ENTRETIEN (RUE ROUSTAING À TALENCE)}

\subsection{Données du site}

Le bassin versant retenu est situé en tête du réseau d'eaux pluviales, de type séparatif. Ses caractéristiques essentielles sont les suivantes :

- aire du bassin versant : $31660 \mathrm{~m}^{2}$

- surface des chaussées : $2400 \mathrm{~m}^{2}$

- pente moyenne : $0,01 \mathrm{~m} / \mathrm{m}$

— trafic : 5000 véh./j

- coefficient de ruissellement : $\mathrm{C}=0,70$

_ débit de pointe attendu sur l'épisode de retour 5 ans : 0,5 $\mathrm{m}^{3} / \mathrm{s}$.

\subsection{Equipement de mesures}

Le regard à l'exutoire du bassin versant, sur le collecteur de $600 \mathrm{~mm}$, a été équipé en poste de mesure des débits et de prélèvements pour analyses qualitatives. Dans ce regard et à proximité ont été installés :

- un déversoir triangulaire,

- deux sondes à ultrasons : une aérienne et une immergée,

- un préleveur automatique programmable d'échantillons,

- un pluviomètre à augets basculeurs,

- un boîtier électrique de gestion et d'acquisition de données.

\subsection{Mode d'accumulation des sédiments}

Sur la chaussée préalablement nettoyée avec la balayeuse aspiratrice habituellement utilisée ont été pratiqués des « nettoyages à blanc " sur des échantillons de surface du caniveau, du bord de chaussée et de l'axe. L'opération a été répétée une, deux et trois semaines plus tard sur des échantillons distincts, sans autre nettoyage, pour quantifier hebdomadairement l'évolution des dépôts. Les sédiments prélevés ont fait l'objet d'analyses granulométriques au laser sur la fraction $0 / 2 \mathrm{~mm}$ et d'analyses physico-chimiques.

Malgré deux épisodes pluvieux qui ont pu perturber la série de mesures initiale, les premières constatations sont les suivantes :

- accumulation des sédiments : décrite dans le tableau 1 ,

- granulométrie des prélèvements : décrite dans le tableau 2.

Cette première série de mesures semble montrer que l'enrichissement se fait par la partie fine $(<20 \mu \mathrm{m})$ de la granulométrie, en particulier sur le bord de la chaussée.

- analyses sur prélèvements : décrite dans le tableau 3.

Tableau 1. - Accumulation des sédiments.

\begin{tabular}{|c|c|c|}
\hline & $\begin{array}{c}\text { masse prélevée } \\
\text { après nettoyage } \\
\text { classique }\left(\mathrm{g} / \mathrm{m}^{2}\right)\end{array}$ & $\begin{array}{c}\text { accumulation en } \\
\text { 1 semaine }\left(\mathrm{g} / \mathrm{m}^{2}\right)\end{array}$ \\
\hline caniveau & 57 & 172 \\
\hline bord de chaussée & 82 & 170 \\
\hline axe & 20 & 82 \\
\hline
\end{tabular}

Tableau 2. - Granulométrie des prélèvements.

\begin{tabular}{|c|c|c|c|c|c|c|c|c|c|}
\hline \multicolumn{2}{|c|}{ PRÉLĖVEMENTS } & \multirow{2}{*}{$\begin{array}{c}\text { Numéro } \\
\text { d'échantillon }\end{array}$} & \multicolumn{7}{|c|}{ PASSANT $(\%)$ AU TAMIS DE : } \\
\hline Situation & Age & & $5 \mu \mathrm{m}$ & $10 \mu \mathrm{m}$ & $20 \mu \mathrm{m}$ & $50 \mu \mathrm{m}$ & $100 \mu \mathrm{m}$ & $500 \mu \mathrm{m}$ & $1000 \mu \mathrm{m}$ \\
\hline \multirow{4}{*}{ Caniveau } & Etat zéro & 1 & 6,8 & 14,1 & 27,7 & 57,5 & 77,5 & 94,3 & 98,1 \\
\hline & +1 semaine & 5 & 5,9 & 11,5 & 20,9 & 40 & 56,8 & 86,3 & 96,1 \\
\hline & +2 semaines & 7 & 5,5 & 10,9 & 18,5 & 32,6 & 46,5 & 78,5 & 94,7 \\
\hline & +3 semaines & 10 & 7,9 & 17,7 & 33,7 & 59,2 & 77,8 & 94,1 & 98,9 \\
\hline \multirow{4}{*}{$\begin{array}{l}\text { Bord } \\
\text { chaussée }\end{array}$} & Etat zéro & 2 & 6 & 12,3 & 24,7 & 52,4 & 70,5 & 91 & 97 \\
\hline & +1 semaine & 6 & 6,3 & 13 & 26,8 & 54,7 & 72,9 & 93,6 & 98 \\
\hline & +2 semaines & 9 & 8,4 & 17,9 & 33,1 & 57,3 & 74,3 & 93,7 & 99,4 \\
\hline & +3 semaines & 13 & 9,2 & 19,8 & 36,5 & 62,7 & 82,3 & 99,2 & 99,8 \\
\hline \multirow{4}{*}{$\begin{array}{l}\text { Axe } \\
\text { chaussée }\end{array}$} & Etat zéro & 3 & 6,5 & 14,1 & 30,9 & 66,4 & 86,6 & 95,4 & 97,2 \\
\hline & +1 semaine & 4 & 6,5 & 13,6 & 28,3 & 58 & 73,7 & 88,2 & 96,5 \\
\hline & +2 semaines & 8 & 4,6 & 9,8 & 20,3 & 44,3 & 62,1 & 82,3 & 95 \\
\hline & +3 semaines & 11 & 9,6 & 22,4 & 43,6 & 69,6 & 80,3 & 84,8 & 92,9 \\
\hline
\end{tabular}


Tableau 3. - Analyses sur prélèvements.

\begin{tabular}{|c|c|c|c|c|c|c|c|c|c|c|c|c|}
\hline \multicolumn{3}{|c|}{ PRÉLĖVEMENTS } & \multicolumn{10}{|c|}{ POLLUTION DES SÉDIMENTS PRÉLEVÉS } \\
\hline Date & Situation & $\mathrm{N}^{*}$ èch. & $\begin{array}{c}\text { M.V. } \\
\%\end{array}$ & $\begin{array}{c}\mathrm{Pb} \\
\mathrm{mg} / \mathrm{kg}\end{array}$ & $\underset{\mathrm{mg} / \mathrm{kg}}{\mathrm{Cu}}$ & $\begin{array}{c}\mathrm{Cd} \\
\mathrm{mg} / \mathrm{kg}\end{array}$ & $\begin{array}{c}\mathrm{Cr} \\
\mathrm{mg} / \mathrm{kg}\end{array}$ & $\begin{array}{c}\mathrm{Ni} \\
\mathrm{mg} / \mathrm{kg}\end{array}$ & $\begin{array}{c}\mathrm{Zn} \\
\mathrm{mg} / \mathrm{kg}\end{array}$ & $\begin{array}{c}\mathrm{Fe} \\
\mathrm{mg} / \mathrm{kg}\end{array}$ & $\begin{array}{c}\mathrm{Al} \\
\mathrm{mg} / \mathrm{kg}\end{array}$ & $\begin{array}{c}\mathrm{Mn} \\
\mathrm{mg} / \mathrm{kg}\end{array}$ \\
\hline \multirow{3}{*}{$\begin{array}{c}\text { 17/06/96 } \\
\text { (état zéro) }\end{array}$} & caniveau & 1 & 16,8 & 1007 & 283 & 10,5 & 70 & 39 & 4998 & 29 & 35,2 & 493 \\
\hline & bord chaussée & 2 & 15,9 & 955 & 218 & 3,5 & 60 & 37 & 2936 & 30,9 & 37,4 & 500 \\
\hline & axe chaussée & 3 & 18,5 & 1209 & 303 & 9,6 & 82 & 59 & 7821 & 43,4 & 42,7 & 629 \\
\hline \multirow{3}{*}{$\begin{array}{c}24 / 06 / 96 \\
(+1 \text { semaine })\end{array}$} & caniveau & 4 & 6,9 & 406 & 129 & 2,5 & 38 & 28 & 1837 & 21,4 & 22,8 & 327 \\
\hline & bord chaussée & 5 & 10,3 & 542 & 148 & 2,4 & 52 & 52 & 1748 & 29,2 & 32,6 & 481 \\
\hline & axe chaussée & 6 & 15,4 & 672 & 179 & 2,2 & 70 & 54 & 2809 & 31,8 & 37,4 & 508 \\
\hline \multirow{3}{*}{$\begin{array}{c}01 / 07 / 96 \\
(+2 \text { semaines })\end{array}$} & caniveau & 7 & 21,4 & 539 & 185 & 4,4 & 48 & 26 & 1853 & 18,3 & 23,4 & 246 \\
\hline & bord chausseee & 8 & 17,2 & 637 & 196 & 3,5 & 53 & 29 & 5619 & 26,9 & 32,2 & 419 \\
\hline & axe chaussée & 9 & 14,6 & 574 & 184 & 2,5 & 61 & 37 & 1884 & 30,1 & 31,7 & 420 \\
\hline \multirow{3}{*}{$\begin{array}{c}08 / 07 / 96 \\
(+3 \text { semaines })\end{array}$} & caniveau & 10 & 16,7 & 797 & 215 & 6,6 & 67 & 35 & 3441 & 31,2 & 36 & 507 \\
\hline & bord chaussée & 13 & 17,1 & 802 & 479 & 10,6 & 106 & 67 & 4422 & 30 & 31,9 & 459 \\
\hline & axe chaussée & 11 & 16,6 & 1213 & 368 & 4,2 & 116 & 71 & 5190 & 53,3 & 45 & 687 \\
\hline
\end{tabular}

Une pluie avant le prélèvement à +1 semaine et une autre avant celui à +2 semaines ont perturbé ces résultats.

On peut toutefois avancer que le balayage classique laisse les particules fines, agglomérées, les plus polluées. Mais ce même balayage reste le minimum nécessaire puisqu'il récupère en masse jusqu'à $80 \%$ environ des sédiments : au point d'avancement actuel de la recherche on ne peut conclure sur le niveau de mobilisation des $20 \%$ restants par le ruissellement.

\subsection{Suivi à l'exutoire}

La figure $l$ illustre la corrélation pluie/débit sur un événement pluvieux et montre que, après un premier pic de pollu- tion (vérifié sur tous les prélèvements effectués), l'entraînement par le ruissellement peut dépendre, entre autres facteurs explicatifs, de l'intensité de la pluie.

\subsection{Poursuite de l'expérimentation}

L'objectif est de :

- mieux cerner le pouvoir de délavage de la pluie, et l'influence de son intensité,

- caractériser physiquement le revêtement de chaussée et essayer de lui associer une loi de stockage-destockage des sédiments.

Le rôle de l'entretien serait alors d'agir sur cette loi pour diminuer la pollution dans le ruissellement.

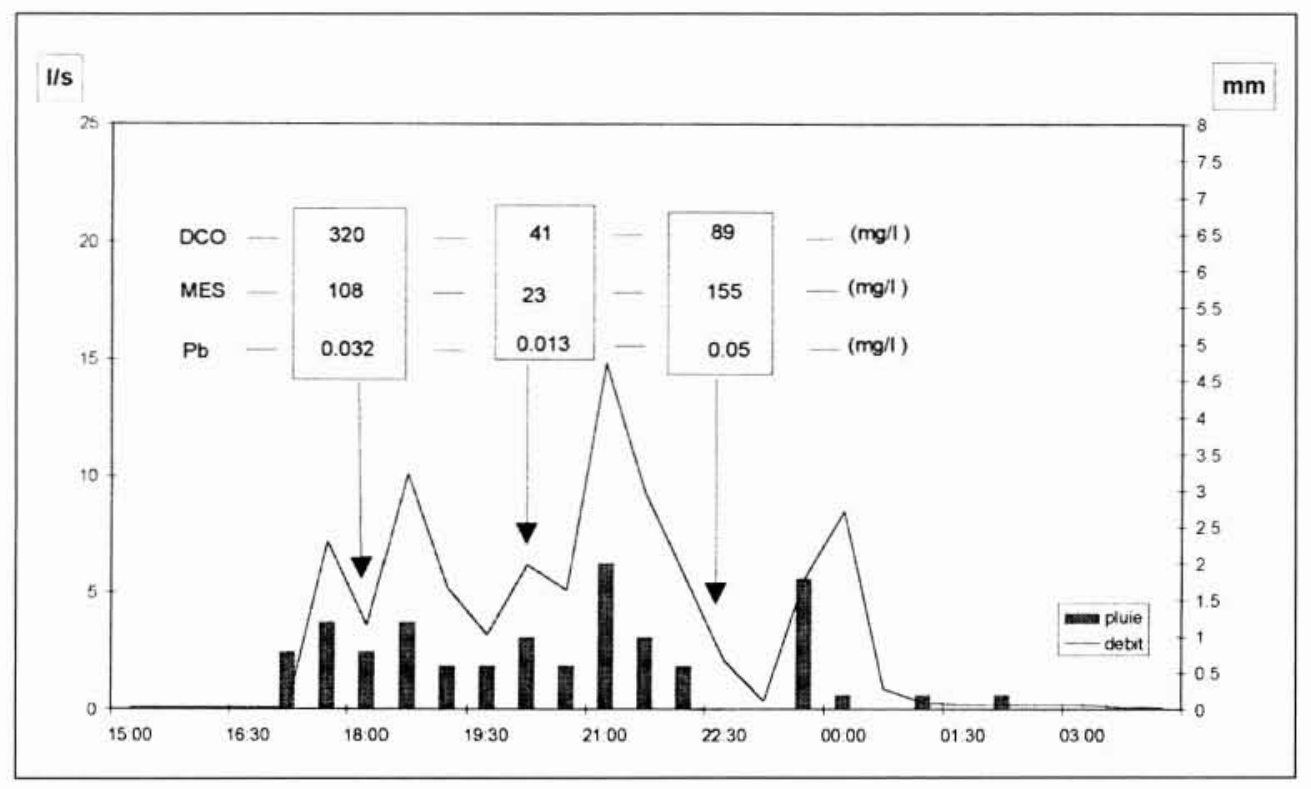

1. Pluie du 04 au 05/11/1996. 


\section{III — IMPACT D'UNE TRANCHÉE DRAI- NANTE (AVENUE GUSTAVE EIFFEL À PESSAC)}

\subsection{Données du site}

Le bassin versant a une superficie de $7540 \mathrm{~m}^{2}$ et correspond à une voirie de zone d'activité, à trafic dense et lourd. Il est situé en tête de réseau séparatif, strictement pluvial, dépourvu d'espaces verts. Il est constitué de :

- une demi-chaussée : $1810 \mathrm{~m}^{2}$

- un parking bétonné : $1270 \mathrm{~m}^{2}$

- la tranchée : $780 \mathrm{~m}^{2}$

— un trottoir non revêtu : $980 \mathrm{~m}^{2}$

— une entreprise : $2700 \mathrm{~m}^{2}$

Caractéristiques :

- coefficient de ruissellement : 0,85

- capacité de la tranchée : $0,9 \mathrm{~m}^{3} / \mathrm{ml}$

- débit de fuite par drain de $250 \mathrm{~mm}$

- réalisation 1990, absence d'entretien.

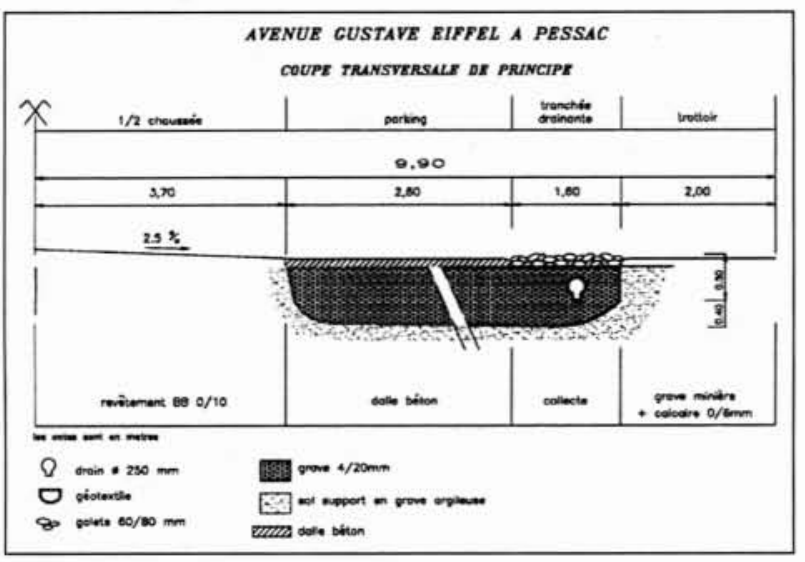

2. Coupe transversale de l'avenue Gustave Eiffel.

\subsection{Equipement de mesures}

A l'exutoire du drain ont été installés les mêmes équipements que pour la rue Roustaing (cf. 2.2). Des piézomètres ont été en outre mis en place pour mesurer les variations de hauteur d'eau dans la tranchée et dans la nappe à proximité.

\section{- 3.3 Prélèvements d'échantillons de sol}

L'objectif de ces prélèvements est de localiser la pollution, de déterminer le devenir des éléments polluants, et notamment leur éventuelle migration vers le sol environnant et la nappe, dans le but de savoir s'il est nécessaire de prévoir des protections ou étanchéités supplémentaires. Une des premières séries de prélèvements a été effectuée en un point situé côté aval de la tranchée (point 2).

Echantillon $n^{\circ} 5$ : dépôt accumulé sur le géotextile, sous les galets $60 / 80$, côté trottoir,

Echantillon $n^{\circ} 6$ : idem, côté parking,

Echantillon $n^{\circ} 7$ : obtenu par lavage des cailloux 4/20 prélevés sur la hauteur de remplissage de la tranchée,

Echantillon $n^{\circ} 8$ : sol en place, au-dessous de la tranchée. La granulométrie des prélèvements est décrite dans le tableau 4.

Les analyses sur prélèvements sont décrites dans le tableau 5.

Les conclusions sont les suivantes:

- la pollution particulaire est piégée à l'entrée du ruissellement dans la tranchée par le géotextile (ex : 459 et $420 \mathrm{mg} / \mathrm{kg}$ de $\mathrm{Pb}$ ) qui joue un rôle de filtre. La pollution non piégée peut se fixer par adsorption sur le granulat drainant (ex : $80 \mathrm{mg} / \mathrm{kg}$ de $\mathrm{Pb}$ ),

- le sol en place est à un niveau de pollution correspondant au bruit de fond (ex : $35 \mathrm{mg} / \mathrm{kg}$ de $\mathrm{Pb}$ ), ce qui semblerait montrer que même les éléments les plus fins sont piégés avant infiltration.

Tableau 4. - Granulométrie des prélèvements.

\begin{tabular}{|c|c|c|c|c|c|c|c|c|}
\hline \multirow{2}{*}{$\begin{array}{c}\text { Situation des } \\
\text { prélèvements }\end{array}$} & \multirow{2}{*}{$\begin{array}{c}\text { Numéro } \\
\text { d'échantillon }\end{array}$} & $5 \mu \mathrm{m}$ & $10 \mu \mathrm{m}$ & $20 \mu \mathrm{m}$ & $50 \mu \mathrm{m}$ & $100 \mu \mathrm{m}$ & $500 \mu \mathrm{m}$ & $1000 \mu \mathrm{m}$ \\
\cline { 2 - 11 } & 5 & 3 & 6 & 11 & 22 & 34 & 73 & 92 \\
\hline \multirow{3}{*}{$\begin{array}{c}\text { AVAL } \\
\text { (point 2) }\end{array}$} & 6 & 4 & 7 & 13 & 25 & 38 & 74 & 95 \\
\cline { 2 - 11 } & 7 & 11 & 19 & 30 & 48 & 60 & 81 & 93 \\
\cline { 2 - 10 } & 8 & 13 & 20 & 27 & 34 & 37 & 58 & 85 \\
\hline
\end{tabular}

Tableau 5. - Analyses sur prélèvements.

\begin{tabular}{|c|c|c|c|c|c|c|c|c|c|c|c|}
\hline Situation & $N^{\circ}$ éch. & M.V. \% & $\mathrm{Pb} \mathrm{mg} / \mathrm{kg}$ & Cu $\mathrm{mg} / \mathrm{kg}$ & $\mathrm{Cd} \mathrm{mg/kg}$ & $\mathrm{Cr} \mathrm{mg} / \mathrm{kg}$ & $\mathrm{Ni} \mathrm{mg} / \mathrm{kg}$ & $\mathrm{Zn} \mathrm{mg/kg}$ & $\mathrm{Fe} \mathrm{mg} / \mathrm{kg}$ & Al $\mathrm{mg} / \mathrm{kg}$ & $\mathrm{Mn} \mathrm{mg} / \mathrm{kg}$ \\
\hline \multirow{4}{*}{$\begin{array}{c}\text { AVAL } \\
\text { (Point 2) }\end{array}$} & 5 & 8,7 & 459 & 76 & 1,28 & 39 & 16 & 298 & 14 & 23 & 189 \\
\hline & 6 & 5 & 420 & 63 & 0,78 & 37 & 17 & 232 & 12 & 17 & 178 \\
\hline & 7 & 2,6 & 80 & 20 & 0,34 & 36 & 23 & 93 & 16 & 47 & 220 \\
\hline & 8 & 2,6 & 35 & 5 & 0,03 & 26 & 9 & 18 & 4 & 47 & 21 \\
\hline
\end{tabular}




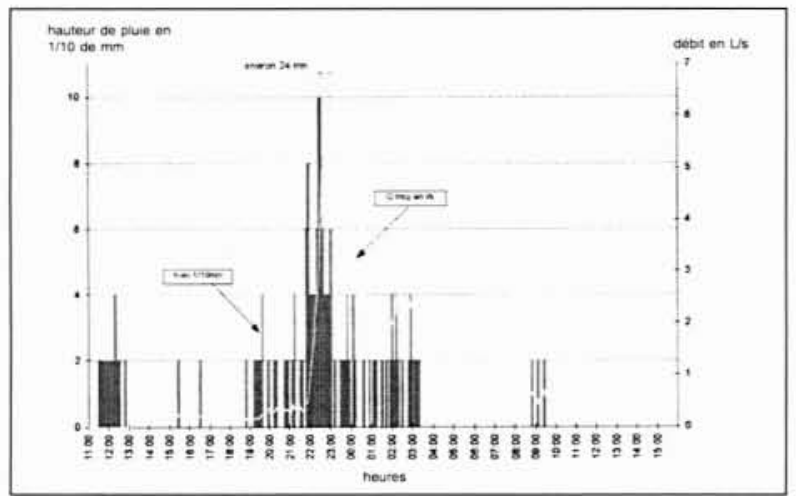

3. Pluie du $10 / 11$ novembre 1996 .

\subsection{Suivi à l'exutoire}

\subsubsection{Comportement hydraulique}

La figure 3 illustre le rôle de stockage de la tranchée, qui diffère les débits ruisselés bien après la pluie. La position haute du drain diminue la capacité de stockage de la tranchée après une période de sollicitation, mais elle favorise sa fonction épuratoire en permettant l'infiltration après retour au temps sec, ou lors de faibles pluies après une période de temps sec. La figure 3 montre à ce sujet qu'il faut plus de $1 \mathrm{~h} 30$ pour solliciter le déversoir (débit non nul) après le début de la pluie.

\subsubsection{Analyses sur prélèvements (tabl. 6)}

Ces résultats montrent l'absence de pollution à l'exutoire, en sortie de drain, confirmant le rôle de filtre du géotextile et traduisant également l'absence de relargage de pollution par le drain : celui-ci ne véhicule donc pas la pollution susceptible de transiter dans les granulats drainants au cours des événements pluvieux.
Tableau 6. - Analyses sur prélèvements.

\begin{tabular}{|c|c|c|c|c|}
\hline \multirow{2}{*}{$\begin{array}{c}\text { PLUIE } \\
\mathrm{N}^{*}\end{array}$} & \multicolumn{4}{|c|}{$\mathrm{mg} / \mathrm{l}$} \\
\cline { 2 - 5 } & DCO & MES & $\mathrm{Pb}$ & $\mathrm{Cd}$ \\
\hline \multirow{3}{*}{1} & 33 & 8 & 0,003 & 0,0008 \\
\cline { 2 - 5 } & 28 & 6 & 0,005 & 0,0005 \\
\cline { 2 - 5 } & 31 & 11 & 0,009 & 0,0005 \\
\hline \multirow{3}{*}{2} & 22 & 7 & 0,004 & 0,0041 \\
\cline { 2 - 5 } & 21 & 9 & 0,005 & 0,0008 \\
\cline { 2 - 5 } & 23 & 15 & 0,011 & 0,0006 \\
\cline { 2 - 5 } & 23 & 13 & 0,011 & 0,0005 \\
\hline
\end{tabular}

\subsection{Poursuite de l'expérimentation}

D'autres prélèvements de sols seront effectués pour s'assurer du devenir des éléments polluants. Il s'agira également de mieux caractériser le fonctionnement hydraulique de la tranchée et de dresser des bilans débits-pollution à l'exutoire, de sorte que cette technique, largement répandue et testée sur la Communauté Urbaine de Bordeaux, pourra être plus finement conçue, dimensionnée et gérée.

\section{IV $\square$ CONCLUSION}

Un des objectifs majeurs du GARIH est la réduction de l'impact du ruissellement urbain. Il répond à ce titre aux préoccupations affichées ces dernières années et il se situe dans la logique des programmes de recherche menés par les organismes centraux comme le LCPC. Le principe de maîtriser la pollution à la source, c'est-à-dire sur la chaussée ou dans son environnement immédiat, se révèle tout à fait pertinent en terme d'efficacité épuratoire : les premiers résultats des études engagées sont encourageants, voire probants, et justifient la démarche entreprise. 